\title{
Asociación entre sentido de coherencia y control glucémico en personas con Diabetes Mellitus tipo 2
}

\author{
Associação entre Senso de Coerência e controle glicêmico em pessoas com Diabetes \\ Mellitus Tipo 2
}

\section{Association between Sense of Coherence and glycemic control in people with Type 2 Mellitus Diabetes}

\author{
Jesús Humberto Márquez-Palacios (iD \\ Universidad Iberoamericana Torreón - Torreón - México \\ José Guadalupe Salazar-Estrada (iD \\ Universidad de Guadalajara (UdG) - Ameca - México
}

Alfonso Urzúa-Morales iD

Universidad Católica del Norte (UCN) - Antofagasta - Chile

\section{RESUMEN}

Objetivo: Evaluar la asociación entre sentido de coherencia y control glucémico en adultos con Diabetes Mellitus tipo 2. Métodos: Estudio de tipo transversal desarrollado durante el año 2018 en el cual fueron encuestados 220 diabéticos de tres unidades de Salud de la Secretaría de Salud del Estado de Jalisco en Ciudad Guzmán, México. Se les aplicó el instrumento SOC-13 para sentido de coherencia y el control glucémico se evaluó por medio del nivel de hemoglobina glucosilada que se obtuvo del expediente médico. Los datos se analizaron por medio del análisis de regresión logística. Resultados: Se obtuvieron resultados con un total de 220 participantes en un rango de edad entre 23 y 69 años de los que el $67 \%$ presentó control glucémico inadecuado y el $33 \%$ control glucémico adecuado; de los encuestados con control glucémico inadecuado el $24 \%$ refirió puntuaciones bajas de sentido de coherencia, mientras que el $96 \%$ de quienes tienen control glucémico adecuado obtuvo puntuaciones medio/alta de sentido de coherencia. Los análisis de regresión logística arrojan que el sentido de coherencia se asocia significativamente con el control glucémico (OR = 7.2; 95\% C.I: 2.0-24.7; $p=0.002)$ después de ajustar los análisis por diversas variables confusoras como sexo, apego al plan alimenticio, actividad física, ausencia de complicaciones y ausencia de tabaquismo. Conclusión: Los diabéticos con puntuaciones medio/altas de sentido de coherencia tienen hasta 7 veces más probabilidad de tener control glucémico adecuado que diabéticos con puntuaciones bajas de sentido de coherencia independientemente de variables intervinientes.

Descriptores: Sentido de Coherencia; Promoción de la Salud; Hemoglobina Glucosilada; Control Metabólico.

\section{RESUMO}

Objetivo: Avaliar a associação entre senso de coerência e controle glicêmico em adultos com diabetes mellitus tipo 2. Métodos: Estudo transversal desenvolvido durante 2018, com 220 diabéticos de três unidades de saúde da Secretaria de Saúde do Estado de Jalisco na cidade de Guzmán, México. Aplicou-se o instrumento SOC-13 para senso de coerência, e avaliou-se o controle glicêmico por meio do nível de hemoglobina glicosada obtido nos prontuários. Assim, obtiveram-se os dados por análise de regressão logística. Resultados: Os 220 participantes tinham faixa etária de 23 a 69 anos, dos quais 148 (67\%) apresentavam controle glicêmico inadequado e 72 (33\%) controle glicêmico adequado; $24 \%$ dos pesquisados com controle glicêmico inadequado relataram escores baixos para senso de coerência, enquanto $96 \%$ daqueles com controle glicêmico adequado obtiveram escores médios/altos para senso de coerência. As análises de regressão logística mostram que o senso de coerência está significativamente associado ao controle glicêmico (OR=7,2; IC 95\%: 2,0-24,7; $p=0,002)$ após ajustar as análises para variáveis de confusão, como sexo, adesão ao plano alimentar, atividade física, ausência de complicações e ausência de tabagismo. Conclusão: Diabéticos, com escores médios/altos de senso de coerência, têm até 7 vezes mais chances de apresentar controle glicêmico adequado do que diabéticos com baixos escores de senso de coerência, independentemente das variáveis intervenientes.

Descritores: Senso de Coerência; Promoção da Saúde; Hemoglobina Glicada; Controle Metabólico. 


\section{ABSTRACT}

Objective: To evaluate the association between a sense of coherence and glycemic control in adults with type 2 Mellitus Diabetes. Methods: Cross-sectional study, developed during 2018, 220 diabetics from three health units of the Secretaria de Salud del Estado de Jalisco in Ciudad Guzmán, Mexico, the SOC-13 instrument was applied for a sense of coherence, glycemic control was evaluated through the level of glycosylated hemoglobin obtained from the medical record, the data was analyzed through logistic regression analysis. Results: Results were obtained with a total of 220 participants in an age range of 23 to 69 years of which $67 \%$ presented inadequate glycemic control and $33 \%$ adequate glycemic control; $24 \%$ of those surveyed with inadequate glycemic control reported low scores for a sense of coherence, while $96 \%$ of those with adequate glycemic control obtained medium/high scores for a sense of coherence. The logistic regression analyzes show that the sense of coherence is significantly associated with glycemic control (OR=7.2; 95\% Cl: 2.0-24.7; $p=0.002)$ after adjusting the analyzes for various confounding variables such as sex, adherence to eating plan, physical activity, absence of complications and absence of smoking. Conclusion: Diabetics with medium/high scores of coherence sense are up to 7 times more likely to have adequate glycemic control than diabetics with low scores of coherence sense regardless of intervening variables.

Descriptors: Sense of Coherence; Health Promotion; Glycated Hemoglobin; Metabolic Control.

\section{INTRODUCCIÓN}

La Diabetes Mellitus (DM) es una enfermedad que afecta a varias personas. Durante 2019 se estimó que en el mundo 1 de cada 11 personas tenía esta enfermedad y los pronósticos indican que para el año 2045 habrá alrededor de 700 millones de personas con $\mathrm{DM}^{(1)}$. La Diabetes Mellitus Tipo 2 (DM2) es el tipo de diabetes más frecuente y ocurre en el $90 \%$ de los casos de diabetes en el mundo(2).

El aumento exponencial de la DM es motivo de atención en todo el mundo debido a que su inadecuado manejo trae consigo la aparición de complicaciones como daños en los nervios periféricos, pie diabético, daños en la retina, daño renal, cardiovasculares ${ }^{(3-6)}$, entre otros, lo que aumenta las tasas de morbilidad y mortalidad ${ }^{(7)}$, así como el costo sanitario asociado a estas ${ }^{(8)}$.

Debido a lo anterior, las ciencias de la salud han enfocado sus esfuerzos en profundizar en el conocimiento sobre la DM y su control para evitar el desarrollo de complicaciones; tal es el caso de la promoción de la salud que se define como el proceso de capacitar a las personas y a las comunidades para que aumenten el control sobre su salud y así poder mejorarla ${ }^{(9)} \mathrm{e}$ implica potenciar estilos de vida saludables y reducir factores de riesgo precursores de enfermedades ${ }^{(10)}$.

Un modelo que ha probado tener buenos resultados en salud es la salutogénesis de la cual se han descrito diversos beneficios en la salud física, mental y en la percepción y calidad de vida de las personas ${ }^{(11)}$. Adicionalmente la salutogénesis y la promoción de la salud guardan diversas similitudes teóricas ${ }^{(12)}$ y se considera que el paradigma salutogénico es un nuevo abordaje en promoción de la salud ${ }^{(13)}$; específicamente con respecto al Control Glucémico (CG) por medio del Sentido de Coherencia (SOC) que es el concepto principal del mencionado paradigma, reportándose hallazgos interesantes ${ }^{(14-18)}$.

EI SOC es una orientación hacia la vida, que expresa hasta qué punto se tiene un sentimiento de confianza dominante, resistente y dinámico, siendo un patrón cognitivo-emocional que refleja la opinión que tiene una persona sobre su propia capacidad para responder ante situaciones consideradas como estresantes y recuperarse de ellas ${ }^{(19)}$.

EI SOC se compone de tres dimensiones: comprensibilidad que hace referencia a la sensación de comprender lo que sucede cuando existe una estimulación ambiental; manejabilidad, definida como la percepción de tener los recursos suficientes para hacer frente a las demandas; y significatividad, que es el componente principal del SOC, debido a que es de tipo motivacional y lleva a las personas a buscar los recursos necesarios para afrontar y solucionar cualquier situación ${ }^{(19)}$.

Puntuaciones altas de SOC se han relacionado a activos en salud, que ayudan a mantener un estado de bienestar. Se ha identificado que el SOC es un factor protector que evita la aparición y el desarrollo de enfermedades ${ }^{(18,20,21)}$. También se asocia al estilo de vida que promueve la salud( ${ }^{(22)}$ y a conductas saludables ${ }^{(23)}$, mayor actividad física ${ }^{(24)}$ y menor consumo de tabaco y alcohol(25,26).

Este constructo también ha sido estudiado en enfermedades crónicas como la diabetes y se documentó que el SOC alto en personas con riesgo a desarrollar DM ayuda a prevenir y/o retardar la aparición de la enfermedad ${ }^{(27-29)}$. También se asocia a un estilo de vida saludable, ya que las personas con SOC alto presentan conductas más 
saludables ${ }^{(14)} y$ tienen mayor probabilidad de cambiar su estilo de vida $(E V)^{(30)}$. Es un hallazgo muy importante debido a que el tratamiento de la DM se centra en el EV con el fin de controlar la enfermedad ${ }^{\left({ }^{11}\right)}$ y evitar el desarrollo de complicaciones $^{(32)}$.

En cuanto al SOC y al control de la enfermedad, también se encontraron resultados alentadores ya que el SOC alto se relaciona con menores niveles de biomarcadores asociados con el control metabólico ${ }^{(14-18,33)}$ y menor incidencia de complicaciones derivadas de la $\mathrm{DM}^{(34)}$. Por lo tanto, el SOC representa una variable protectora para la prevención, el control y el desarrollo de la DM así como también evita el desarrollo de complicaciones.

Sin embargo, en un estudio reciente de revisión sistemática ${ }^{(35)}$, que analizó el efecto del SOC en personas con diabetes, los investigadores identificaron que en algunos estudios la medición del SOC y los biomarcadores no se realizaron a la par. Esta situación podría sesgar los resultados, debido a que la hemoglobina glucosilada (HbA1c) muestra el nivel promedio de glucosa de los últimos tres meses ${ }^{(1)}$ y en el caso del SOC se ha documentado que aumenta con la edad ${ }^{(36,37)}$. Por lo tanto, en este ejemplo si transcurren más de tres meses entre la medición de la $\mathrm{HbA} 1 \mathrm{c}$ y el SOC los niveles de una o ambas variables pudieron cambiar y la asociación entre las mismas podría ser diferente a lo reportado; en ello radica la importancia de la medición conjunta de las variables clínicas y psicológicas para evitar este tipo de sesgos.

Así pues, esta investigación tuvo el objetivo de evaluar la asociación entre sentido de coherencia y control glucémico en adultos con Diabetes Mellitus tipo 2.

\section{MÉTODOS}

Se trata de una investigación de corte transversal del tipo asociativa desarrollada durante el año 2018.

El universo poblacional lo conformaron personas de ambos sexos mayores de 20 y hasta los 69 años de edad que tuvieran el diagnóstico previo de DM2 y estuvieran recibiendo tratamiento para el control de la enfermedad en el Hospital Regional de Ciudad Guzmán, el Centro de Salud \#1 y/o el Centro de Salud \#2 pertenecientes a la Secretaría de Salud en Ciudad Guzmán, Jalisco, México.

El tamaño de la muestra calculado se estableció en 210 personas, pero se aumentó a 242. Este $15 \%$ extra fue debido a que se estima que alrededor de $10 \%$ de los diabéticos tiene DM tipo 1 . Además, se consideró una tasa de no respuesta del 5\%; el tamaño de la muestra fue calculado por medio del programa Epidat 3.1 y la técnica de muestreo aplicada fue por conveniencia.

Se incluyeron ambos sexos en edades entre 20 y 69 años, diagnosticados con DM2, en tratamiento y con resultados de $\mathrm{HbA} 1 \mathrm{c}$ con vigencia máxima de siete días. Fueron excluidos aquellos cuestionarios que no tenían contestados $100 \%$ de los ítems y/o que no tengan los resultados clínicos de la glucosa en sangre y/o que no desearon seguir participando en la investigación.

Para medir el SOC se usó la escala de Orientación a la Vida en español versión 13 ítems (SOC-13). En cuanto a sus propiedades psicométricas, la confiabilidad ha sido reportada en previas aplicaciones en el contexto latinoamericano con valores alfa de Cronbach que oscilan entre 0.79 y 0.80 , así como la estructura factorial que propone el autor original del instrumento ${ }^{(38)}$.

Para obtener los resultados de la HbA1c, se recurrió a consultar el expediente médico del paciente.

Se reclutaron a pacientes ambulatorios en el Hospital Regional de Ciudad Guzmán, del Centro de Salud\#1 y Centro de Salud \#2 de la misma localidad. Los pacientes fueron abordados mientras se encontraban en la sala de espera. Primero se indagaba si los posibles participantes cumplían con los criterios de inclusión. En caso de respuesta afirmativa se les solicitaba su autorización por medio de un consentimiento informado para participar en el estudio. Posteriormente se aplicaba la ficha sociodemográfica y el cuestionario. Respecto al análisis clínico se solicitaba el número del expediente para obtener el dato. La técnica de muestreo fue por conveniencia, por lo tanto, se trabajó en las unidades de salud mencionadas hasta que se completó el tamaño de la muestra. La tasa de rechazo fue del $5 \%$ del total de la muestra. El llenado de la ficha sociodemográfica y el cuestionario les tomó a los participantes aproximadamente 10 minutos.

Para las clasificaciones todas las variables fueron dicotomizadas. EI CG fue determinado por medio de la HbA1c y se consideraron los siguientes criterios para clasificarla: control glucémico inadecuado $\mathrm{HbA} 1 \mathrm{c}>7 \%$; mientras que el control glucémico adecuado $\mathrm{HbA} 1 \mathrm{c}<=7 \%$, esto conforme a diversos estudios que sugieren el uso de los anteriores puntos de corte ${ }^{(39,40)}$. Una vez dicotomizada la variable dependiente, a las personas con CG inadecuado se les asignaba el 0 y a las personas con CG adecuado el 1 para la ejecución de la regresión logística. 
En cuanto al sentido de coherencia, se dividió a la población en dos categorías, SOC bajo y SOC medio/alto. Las personas que obtuvieran una puntuación total en la escala SOC-13 menor o igual a 44 fueron categorizadas con SOC bajo mientras que puntuaciones iguales o superiores a 45 fueron identificadas con SOC medio/alto. Para obtener estos puntos de corte se realizó lo siguiente: se obtuvo la puntuación total de SOC de cada participante, después se calculó la media y la desviación estándar de la puntuación total de SOC considerando a todos los participantes. Una vez definida la media y la desviación estándar se restó una desviación estándar a la media para establecer la puntuación baja (SOC bajo) y los valores que fueran superiores por un punto a la categoría SOC bajo fueron considerados como SOC medio/alto. En cuanto al análisis estadístico, las personas con SOC bajo fueron identificadas con el número 0 y las personas con SOC medio/alto con el número 1.

Con respecto a las variables que fueron posibles factores de confusión, sexo $(0=$ mujer; $1=$ hombre $)$, ausencia de complicaciones $(0=\mathrm{No} ; 1=\mathrm{Sí})$ y ausencia de tabaquismo $(0=\mathrm{No} ; 1=\mathrm{Sí})$ son dicotómicas, mientras que para dicotomizar las variables actividad física $(0=$ No; $1=$ Sí $)$ y apego al plan alimenticio $(0=$ No; $1=$ Sí $)$ se hizo el siguiente procedimiento: la inactividad física se evaluó con la pregunta "¿En cuántos de los últimos siete días usted participó en una sesión de ejercicios específicos (ejemplo: nadar, caminar, andar en bicicleta) que no sea lo que se hace en la casa o como parte de su trabajo?", si la persona respondía entre cero y dos días de los últimos siete se categorizaba con el número cero, es decir inactivo, mientras que si respondía entre tres y siete cualquier opción se etiquetaba con el número uno, es decir activo; mientras que la variable apego al plan alimenticio se evaluó con la pregunta "En el último mes, ¿cuántos días a la semana usted ha seguido el plan de alimentación?", resultados entre cero y dos días se categorizaban con el número cero, es decir no sigue el plan y respuestas entre tres y siete días se consideraban como que sí seguían el plan de alimentación y se identificaban con el número uno.

En la primera fase se obtuvieron estadísticos descriptivos como medidas de tendencia central, dispersión y frecuencias con el fin de caracterizar socio demográficamente a la población de estudio. Dichos análisis fueron ejecutados por el programa estadístico Statistical Package for the Social Sciences (SPSS) versión 25 para Windows.

En la segunda fase se analizaron las propiedades psicométricas de la escala SOC-13. Se analizó la consistencia interna mediante el coeficiente de Alfa de Cronbach $(\alpha)$ de forma global calculado por medio del programa SPSS. También fue evaluada la validez de constructo por medio del análisis factorial confirmatorio (AFC) a través del estimador Weighted Least Squares Means and Variance Adjusted (WLSMV). Se consideraron los siguientes índices: el índice de ajuste comparativo (CFI de 0.90 a 0.95 aceptable) $)^{(41)}$, el error cuadrático medio de aproximación (RMSEA de 0.06 a 0.08 aceptable) $)^{(42)}$ y el índice de Tucker Lewis (TLI $\geq 0.90$ aceptable) $)^{(43)}$. La determinación de la validez de constructo fue calculada con el programa MPlus versión 7.3.

Por último, en la tercera fase con las variables dicotomizadas se realizó la regresión logística para calcular el odds ratio de prevalencia. Fue considerado un intervalo de confianza del 95\%. De esta forma se asoció el CG y el SOC ajustado por posibles factores de confusión (sexo, apego al plan alimenticio, actividad física, ausencia de complicaciones y ausencia de tabaquismo). Se consideró una asociación estadísticamente significativa cuando el resultado del intervalo de confianza no incluyó al ${ }^{1(44)}$. Este análisis se realizó de la siguiente forma: el CG fue la variable dependiente, mientras que el SOC fue la variable independiente. También se incluyeron las variables sexo, apego al plan alimenticio, actividad física, ausencia de complicaciones y ausencia de tabaquismo como variables confusoras. Se realizó el cálculo de la asociación de cada una de estas variables con la variable dependiente con el objetivo de ajustar el efecto de la variable independiente sobre la dependiente y así reducir la posibilidad de mostrar relaciones espurias. Los análisis estadísticos fueron ejecutados en el programa estadístico SPSS versión 25 para Windows.

La investigación ha sido registrada y aprobada por el Comité de Bioética del Centro Universitario del Sur de la Universidad de Guadalajara con la resolución CB/022/2017.

\section{RESULTADOS}

La muestra total fue de 220 personas y las características sociodemográficas de la población son las siguientes:164 (74.5\%) eran mujeres mientras que solo $56(25.5 \%)$ fueron hombres y el rango de edad de los participantes fue de 23 a 69 años. Más de la mitad de la muestra estaba casada 125 (56.8\%) y solo tenía estudios de educación básica, específicamente primaria 144 (65.5\%), también 143 (65\%) era desempleada.

Respecto a los factores que inciden sobre el CG, la mayoría $(n=194 ; 88.2 \%)$ no presentaban complicaciones agudas de la diabetes. Solo $26(11.8 \%)$ dijeron tener complicaciones agudas (hipoglucemia y/o hiperglucemia y/o cetosis y/o cetoacidosis y/o coma hiperosmolar) mientras que el $100 \%$ de la muestra refirió no tener complicaciones crónicas (daños en los nervios y/o la retina y/o renal y/o cardiovasculares y/o pie diabético y/o esteatosis hepática y/o dermopatía diabética). En cuanto al tabaquismo, 208 (94.5\%) no eran fumadores y $12(5.5 \%)$ refirieron que 
sí fuman; sobre la actividad física, 70 (31.8\%) mencionaron estar activos físicamente en la última semana y 150 $(68.2 \%)$ dijeron ser sedentarios y no hacer actividad física en los últimos siete días. Por último, 28 (12.7\%) personas mencionaron que han seguido el plan alimenticio recomendado por los profesionales de la salud en el último mes y $192(87.3 \%)$ no han seguido estas recomendaciones dietéticas.

Respecto las características clínicas de la población, se identificaron un total de 148 (67\%) pacientes con CG inadecuado, mientras que $72(33 \%)$ tuvieron valores de glucosa en sangre menores o iguales al $7 \%$ lo que los caracteriza como diabéticos con CG adecuado. La distribución de las variables sociodemográficas y la variable SOC en personas con CG inadecuado y adecuado, se muestra en la Tabla I.

Tabla I - Descripción de la población de estudio. Ciudad Guzmán, Jalisco, México. 2018.

\begin{tabular}{|c|c|c|}
\hline Variables & Control adecuado & Control inadecuado \\
\hline \multicolumn{3}{|l|}{ Sexo n (\%) } \\
\hline Mujer & $57(79,2)$ & $107(72,3)$ \\
\hline Hombre & $15(20,8)$ & $41(27,7)$ \\
\hline \multicolumn{3}{|c|}{ Estado Civil, n (\%) } \\
\hline Soltero & $17(23,6)$ & $32(21,6)$ \\
\hline Casado & $41(56,9)$ & $84(56,8)$ \\
\hline Unión Libre & $2(2,8)$ & $9(6,1)$ \\
\hline Divorciado & $3(4,2)$ & $6(4,1)$ \\
\hline Viudo & $9(12,5)$ & $17(11,5)$ \\
\hline \multicolumn{3}{|c|}{ Escolaridad n (\%) } \\
\hline Primaria & $47(65,3)$ & $97(65,5)$ \\
\hline Secundaria & $11(15,3)$ & $34(23)$ \\
\hline Preparatoria & $9(12,5)$ & $10(6,8)$ \\
\hline Universidad & $5(6,9)$ & $7(4,7)$ \\
\hline \multicolumn{3}{|l|}{ Ocupación n (\%) } \\
\hline Desempleado & $49(68,1)$ & $94(63,5)$ \\
\hline Empleado & $18(25)$ & $35(23,6)$ \\
\hline Comerciante & $5(6,9)$ & $18(12,2)$ \\
\hline Jubilado & $0(0)$ & $1(0,7)$ \\
\hline \multicolumn{3}{|c|}{ Ausencia de complicaciones $\mathrm{n}(\%)$} \\
\hline Sí & $65(90,3)$ & $129(87,2)$ \\
\hline No & $7(9,7)$ & $19(12,8)$ \\
\hline \multicolumn{3}{|c|}{ Actividad física n (\%) } \\
\hline Sí & $30(41,7)$ & $40(27)$ \\
\hline No & $42(58,3)$ & $108(73)$ \\
\hline \multicolumn{3}{|c|}{ Apego al plan de alimentación $n(\%)$} \\
\hline Sí & $5(6,9)$ & $25(16,9)$ \\
\hline No & $67(93,1)$ & $123(83,1)$ \\
\hline \multicolumn{3}{|c|}{ Ausencia de tabaquismo n (\%) } \\
\hline Sí & $69(95,8)$ & $139(93,9)$ \\
\hline No & $3(4,2)$ & $9(6,1)$ \\
\hline \multicolumn{3}{|c|}{ Sentido de Coherencia n (\%) } \\
\hline Bajo & $3(4,2)$ & $36(24,3)$ \\
\hline Medio/Alto & $69(95,8)$ & $112(75,7)$ \\
\hline
\end{tabular}

Respecto a las propiedades psicométricas de la escala SOC-13, la Tabla II presenta los indicadores globales de ajuste del análisis factorial confirmatorio. Fue necesario reespecificar el modelo para mejorar el ajuste global. Dicho ajuste se alcanzó después de correlacionar los ítems 2 con 3, 2 con 5 así como 3 con 5 . Estos ítems corresponden a las dimensiones de comprensibilidad (ítem 2) y manejabilidad (ítems 3 y 5). Posterior a esto el ajuste global del modelo fue óptimo, indicando que es una buena representación de la composición factorial de la escala. En la Figura 1 se muestra la estructura factorial por ítems y sus respectivas dimensiones. 
Tabla II - Indicadores de ajuste global del análisis factorial confirmatorio. Ciudad Guzmán, Jalisco, México. 2018.

\begin{tabular}{lcccccccc}
\hline \multirow{2}{*}{ Análisis factorial confirmatorio } & \multirow{2}{*}{$\mathbf{X}^{2}$} & d.f & $\mathbf{p}$ & \multirow{2}{*}{ CFI } & \multirow{2}{*}{ TLI } & \multirow{2}{*}{ RMSEA } & \multicolumn{2}{c}{ RMSEA CI 90\% } \\
\cline { 5 - 9 } & 141,411 & 59 & 0,000 & 0,925 & 0,900 & 0,080 & 0,063 & 0,097 \\
\hline Modelo de medición & 14,0 & Alto \\
\hline
\end{tabular}

$X^{2}$ : chi cuadrado. d.f: grados libertad. p: significancia. CFI: índice de ajuste comparativo. TLI: índice de Tucker Lewis. RMSEA: error cuadrático medio de aproximación. Cl: intervalo de confianza. Fuente: elaboración propia

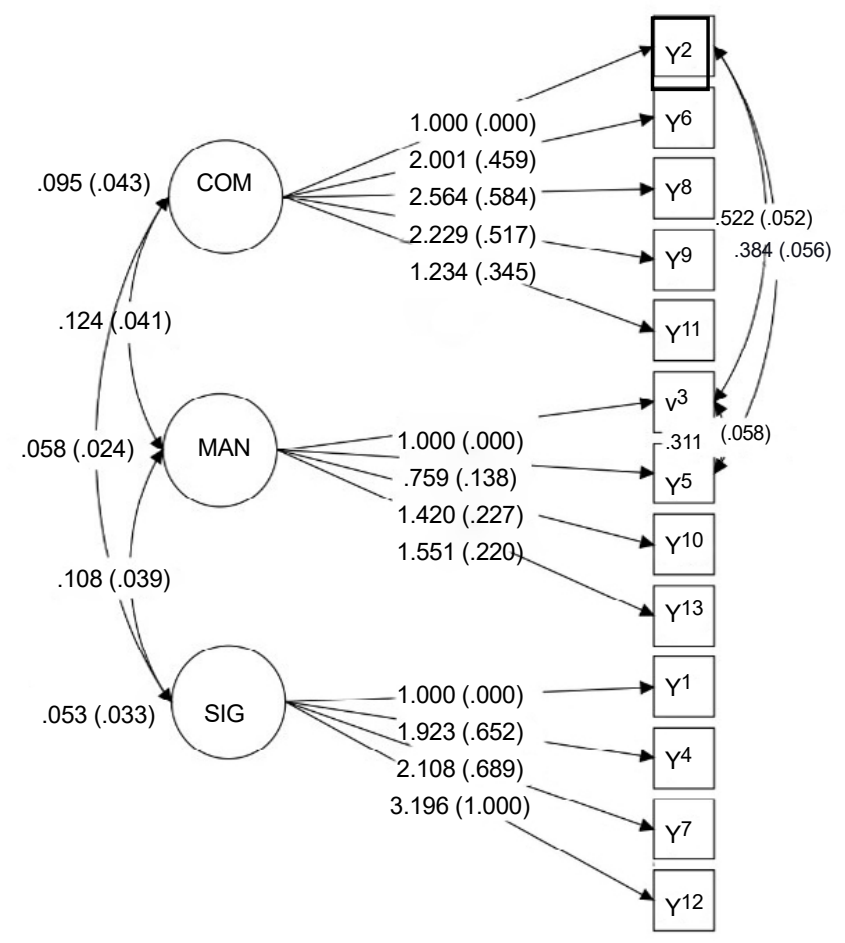

COM: Comprensibilidad; MAN: Manejabilidad; SIG: Significatividad. Ítems de la escala del y1 al y13. Fuente: elaboración propia Figura 1 - Análisis factorial confirmatorio de trece ítems agrupados en tres dimensiones. Ciudad Guzmán, Jalisco, México. 2018.

En lo que concierne a la relación entre SOC y CG, la asociación por medio de la regresión logística muestra que el SOC se asocia con el CG de forma significativa (OR=7.2; 95\% C.I: $2.0-24.7 ; p=0.002)$ lo que nos dice que la razón entre diabéticos con CG inadecuado versus CG adecuado es 7 veces mayor en diabéticos con puntuación medio/ alta de SOC en comparación a puntuación baja de SOC (después de ajustar por diversos factores confusores: sexo, apego al plan alimenticio, actividad física, ausencia de complicaciones y ausencia de tabaquismo). En la Tabla III se pueden consultar los resultados de todas las variables asociadas con la variable dependiente.

Tabla III - Coeficientes de regresión logística. Ciudad Guzmán, Jalisco, México. 2018.

\begin{tabular}{lccccc}
\hline \multirow{2}{*}{ Regresión logística } & \multirow{2}{*}{ Beta } & Sig & \multicolumn{3}{c}{ OR } \\
\cline { 5 - 6 } & & & Valor & Inf & Sup \\
\hline Sexo & 0,432 & 0,226 & 1,54 & 0,763 & 3,14 \\
$\quad$ Apego al plan de alimentación & 0,886 & 0,131 & 2,42 & 0,769 & 7,65 \\
Actividad física & 0,465 & 0,156 & 1,59 & 0,837 & 3,02 \\
Ausencia de complicaciones & 1,97 & 0,482 & 1,41 & 0,537 & 3,72 \\
Ausencia de tabaquismo & $-0,037$ & 0,960 & 0,964 & 0,230 & 4,04 \\
Sentido de Coherencia & 1,95 & 0,002 & 7,20 & 2,09 & 24,73 \\
\hline
\end{tabular}

Sig: significancia. OR: odds ratio. Inf: inferior. Sup: superior. Fuente: elaboración propia 


\section{DISCUSIÓN}

Los resultados indican que los diabéticos con puntuación medio/alto de SOC tendrán hasta 7 veces más probabilidades de tener CG adecuado con respecto a los diabéticos con puntuaciones bajas de SOC, independientemente de variables intervinientes como el sexo, el apego al plan alimenticio, la actividad física, la ausencia de complicaciones y la ausencia de tabaquismo. Estos resultados son acordes con estudios previos ${ }^{(14-17)}$.

Se consideró que estos resultados en parte se deben a que las personas con SOC alto tienen elecciones saludables de estilo de vida independientemente de la clase social y la educación ${ }^{(26)}$, tienen mejores hábitos dietéticos y elecciones alimenticias saludables ${ }^{(23)}$, y se sabe que el puntaje de SOC tiene una estrecha relación con las concepciones saludables de peso corporal y alimentación ${ }^{(45)}$. Así pues, el SOC alto promueve la salud a través de la adopción de un mejor estilo de vida y hábitos alimenticios saludables que ayudan a mantener un peso adecuado. Estos beneficios en alimentación y peso corporal facilitan que los diabéticos tengan un control glucémico adecuado $^{(46)}$ que se considera por abajo del $7 \%$ en $\mathrm{HbA} 1 \mathrm{c}$.

En esta investigación fueron tomados en cuenta diversos aspectos metodológicos señalados en investigaciones previas $^{(35)}$ que podrían sesgar los resultados. Para evitar este sesgo, se atendieron las siguientes recomendaciones: la más importante fue que la medición del SOC y los biomarcadores se haga en un corto periodo de tiempo (máximo 7 días). Se consideró que este criterio fue omitido en estudios anteriores ${ }^{(14-17)}$ debido a que, en los inicios de la teoría salutogénica un estudio afirmaba que el SOC era estable y se desarrollaba en la edad adulta culminando alrededor de los 30 años ${ }^{(47)}$. Por tal motivo, después de esta edad se creía que el nivel de SOC era consistente a través del tiempo. Sin embargo, investigaciones recientes afirman que el SOC cambia a través de los años debido a las experiencias vividas y algunas intervenciones pueden contribuir a modificar el nivel de SOC en las personas ${ }^{(11,48)}$. Por este motivo se consideró importante medir a la par los biomarcadores y el SOC para evitar que los cambios en los niveles de una o ambas variables debido al paso del tiempo intervenga en las asociaciones.

Otra recomendación, fue considerar la posible influencia de variables distintas al SOC sobre el nivel de HbA1c. Se consideraron variables como: sexo $^{(49)}$, apego al plan alimenticio ${ }^{(50)}$, actividad física ${ }^{(51)}$, ausencia de complicaciones ${ }^{(52)}$ y ausencia de tabaquismo ${ }^{(53)}$, debido a que son variables que influyen en el nivel de glucosa en la sangre. Se recomienda, sobre todo, en estudios de corte epidemiológico, considerar en el análisis estadístico variables que podrían afectar a la variable dependiente para no mostrar conclusiones espurias ${ }^{(54)}$. Por último, se consideró realizar la medición del SOC por medio de un instrumento válido y confiable en la población diana ya que se ha reportado que existe una versión del instrumento para medir SOC que no tiene confiabilidad aceptable ${ }^{(55)}$.

Sobre la medición del SOC se usó la versión SOC-13 del Cuestionario de Orientación a la Vida ${ }^{(19)}$ que ha sido adaptada al español(56) y cuenta con evidencia de confiabilidad y validez ${ }^{(38)}$. En el presente estudio se obtuvieron los siguientes datos: la confiabilidad se aseguró por medio del Alfa de Cronbach, el índice fue de 0.78 para la escala general, valor que se encuentra dentro del rango recomendado de 70 a $95^{(57)}$.

La validez de constructo se evaluó por medio del AFC y los índices de bondad de ajuste son los siguientes $\mathrm{CFI}=0.92, \mathrm{TLI}=0.90, \mathrm{RMSEA}=0.08$, que contrastados con los recomendados en la literatura son bastante aceptables: CFI mayor a $0.90^{(41)}$, TLI mayor a $0.90^{(43)}$ y RMSEA entre 0.06 y $0.08^{(42)}$. La estructura factorial puede ser apreciada en la figura 1, si bien, en esta aplicación se tuvo que reespecificar el modelo correlacionando algunos ítems ( 2 con 3 y 5 , así como 3 con 5). Dichas correlaciones tienen sentido teórico, debido a que los ítems corresponden a las dimensiones de comprensibilidad y manejabilidad y diversos estudios consideran estas dos dimensiones en una sola ${ }^{(58,59)}$. Las correlaciones se realizaron para alcanzar adecuados valores de bondad de ajuste.

Se consideró que esto puede tener su origen en que la escala SOC-13 fue validada en estudiantes universitarios y en este estudio se aplicó en población clínica mayor de 23 años. Posiblemente la adaptación de la escala requiera realizar algunos ajustes en la población de este estudio para replicar de forma exacta la estructura original. No obstante, la estructura factorial de tres factores guarda la misma distribución de ítems en cada factor que la versión validada $^{(38)}$. Los valores de bondad de ajuste y estructura interna son similares a los de la escala original ${ }^{(38)}$, por lo tanto, la medición en este estudio del SOC se ha realizado con un instrumento válido y confiable.

Las fortalezas de esta investigación fueron evaluar el SOC y la HbA1c con menos de siete días de diferencia entre ambas mediciones, también incluir en la regresión logística variables que pueden modificar el nivel de HbA1c en los diabéticos y medir el SOC con una versión validada previamente. Todos estos elementos ayudan a tener resultados con más objetividad y reafirman el rol del SOC en el control glucémico después de controlar estos errores metodológicos señalados en estudios anteriores ${ }^{(35)}$. Las debilidades del estudio son el tamaño de la muestra ya que una muestra más grande hubiera permitido una mayor robustez en el modelo y la técnica del muestreo. Consideramos que un muestreo aleatorio simple hubiera permitido extrapolar los resultados. 
Se recomienda en futuras investigaciones que se incluya al SOC como una tercer variable (o variable $Z$ ) en la relación entre una variable independiente (variable $X$ ) y una dependiente (variable de respuesta $Y$ ), lo que ayudaría a profundizar en la comprensión del modelo salutogénico con respecto a las enfermedades crónicas y en especial a la DM, debido a que este tipo de análisis nos ayuda a comprender los procesos y mecanismos causales por los que modelos como el salutogénico influyen en el bienestar. También consideramos que realizar estudios experimentales aportaría más evidencia científica de alta calidad metodológica para lograr comprender el papel del SOC en la DM. En la aplicación práctica de la promoción de la salud se recomienda que se considere la inclusión del modelo salutogénico para el desarrollo de programas preventivos y de intervención para la mejora de adherencia al tratamiento y modificación de estilos de vida no solo en personas con diabetes sino con cualquier enfermedad crónica no transmisible que necesite de la adopción de hábitos saludables.

\section{CONCLUSIÓN}

El modelo salutogénico contribuye al mantenimiento y desarrollo de la salud, la evidencia científica sobre la salutogénesis, brinda un conocimiento detallado de la efectividad del modelo y demuestra su potencial en la investigación como una construcción positiva y promotora de la salud, donde a medida que se tenga un sentido de coherencia más alto se realizará un mejor uso de los recursos que ayudarán a promover la salud. En personas con DM2 el SOC confirma ser un constructo promotor de la salud, reafirma su carácter de factor protector para el adecuado control del azúcar en sangre y con ello evitar el desarrollo de complicaciones diabéticas.

\section{CONFLICTOS DE INTERÉS}

Los autores de este trabajo declaran no tener conflictos de interés.

\section{CONTRIBUCIONES}

Jesús Humberto Márquez-Palacios contribuyó a la preparación y diseño del estudio; la adquisición, análisis e interpretación de datos; y la redacción y/o revisión del manuscrito. Alfonso Urzúa-Morales contribuyó a la preparación y diseño del estudio; análisis e interpretación de datos; y revisión del manuscrito. José Salazar-Estrada contribuyó al análisis e interpretación de datos y la redacción y / o revisión del manuscrito.

\section{AGRADECIMIENTOS}

Al Consejo Nacional de Ciencia y Tecnología por su apoyo para realizar esta investigación por medio de la beca Nro. 465514.

Manuscrito basado en la tesis de doctorado intitulada: "Sentido de coherencia, autocuidado, empoderamiento y glucemia en personas con diabetes", año 2020, 135 páginas.

\section{FUENTES DE FINANCIAMIENTO}

Beca de Doctorado del Consejo Nacional de Ciencia y Tecnología (CONACyT).

\section{REFERENCIAS}

1. Federación Internacional de Diabetes. Atlas de la Diabetes 2019 [Internet]. 9a ed. Bruxelas, BE: Federación Internacional de Diabetes; 2019 [citado el 2018 Jan 28]. Disponible en: https://diabetesatlas.org/es/resources

2. American Diabetes Association. Classification and Diagnosis of Diabetes: Standards of Medical Care in Diabetes - 2020. Diabetes Care. 2020;43(Supplement 1):S14-31.

3. Corona G, Giorda CB, Cucinotta D, Guida P, Nada E. Sexual Dysfunction in Type 2 Diabetes at Diagnosis: Progression over Time and Drug and Non-Drug Correlated Factors. PLoS ONE [Internet]. 2016 [citado el 2018 Jan 28];11(10). Disponible en: https://www.ncbi.nlm.nih.gov/pmc/articles/PMC5051725/

4. Herman WH, Ye W, Griffin SJ, Simmons RK, Davies MJ, Khunti K, et al. Early Detection and Treatment of Type 2 Diabetes Reduce Cardiovascular Morbidity and Mortality: a Simulation of the Results of the Anglo- 
Danish-Dutch Study of Intensive Treatment in People With Screen-Detected Diabetes in Primary Care (ADDITION-Europe). Diabetes Care. 2015;38(8):1449-55.

5. Nithyaa AN, Premkumar R, Dhivya S, Vennila M. A Real Time Foot Pressure Measurement for Early Detection of Ulcer Formation in Diabetics Patients Using Labview. Procedia Eng. 2013;64:1302-9.

6. Olafsdottir E, Andersson DKG, Dedorsson I, Svärdsudd K, Jansson SPO, Stefánsson E. Early detection of type 2 diabetes mellitus and screening for retinopathy are associated with reduced prevalence and severity of retinopathy. Acta Ophthalmol (Copenh). 2016;94(3):232-9.

7. Wacher NH, Gómez-Díaz RA, Casas-Saavedra LP. Causas de descontrol metabólico en pacientes con diabetes en atención primaria en el Instituto Mexicano del Seguro Social (IMSS). Rev Investig Clínica. 2014;66(4):369-78.

8. Bommer C, Heesemann E, Sagalova V, Manne-Goehler J, Atun R, Bärnighausen T, et al. The global economic burden of diabetes in adults aged 20-79 years: a cost-of-illness study. Lancet Diabetes Endocrinol. 2017;5(6):423-30.

9. Ottawa Charter. Ottawa Charter for Health Promotion. Health Promot Int. 1986;1(4).

10. Sánchez PT, Jiménez MJ, Montesinos BL. La promoción de la Actividad Física orientada hacia la salud: un camino por hacer. Eur J Hum Mov. 1998(4):203-17.

11. Mittelmark MB, Sagy S, Eriksson M, Bauer GF, Pelikan JM, Lindström B, et al., editores. The Handbook of Salutogenesis [Internet]. Cham, CH: Springer; 2017 [citado el 2018 Feb 23]. Disponible en: http://www.ncbi. nlm.nih.gov/books/NBK435831/

12. Eriksson M, Lindström B. A salutogenic interpretation of the Ottawa Charter. Health Promot Int. 2008 Jun;23(2):190-9.

13. Ramones EMR. Camino salutogénico: estilos de vida saludable. Rev Digit Postgrado [Internet]. 2019 [citado el 2021 Jun 17];8(1). Disponible en: http://portal.amelica.org/ameli/jatsRepo/101/101599007/html/

14. Ahola AJ, Mikkilä V, Saraheimo M, Wadén J, Mäkimattila S, Forsblom C, et al. Sense of coherence, food selection and leisure time physical activity in type 1 diabetes. Scand J Public Health. 2012 Nov;40(7):621-8.

15. Ahola AJ, Saraheimo M, Forsblom C, Hietala K, Groop P-H, FinnDiane Study Group. The cross-sectional associations between sense of coherence and diabetic microvascular complications, glycaemic control, and patients' conceptions of type 1 diabetes. Health Qual Life Outcomes. 2010 Nov;8:142.

16. Nuccitelli C, Valentini A, Caletti MT, Caselli C, Mazzella N, Forlani G, et al. Sense of coherence, self-esteem, and health locus of control in subjects with type 1 diabetes mellitus with/without satisfactory metabolic control. J Endocrinol Invest. 2018 Mar;41(3):307-14.

17. Linden K, Sparud-Lundin C, Adolfsson A, Berg M. Well-Being and Diabetes Management in Early Pregnant Women with Type 1 Diabetes Mellitus. Int J Environ Res Public Health. 2016;13(8).

18. Olesen K, Jensen TM, Diaz LJ, Møller ACL, Willaing I, Lyssenko V. Sense of Coherence is associated with LDL-cholesterol in patients with type 1 diabetes - The PROLONG-Steno study. J Clin Transl Endocrinol. 2017 Jun;8:1-5.

19. Antonovsky A. Unraveling the mystery of health: how people manage stress and stay well. [lugar desconocido]: Jossey-Bass; 1987. 248 p.

20. Ograczyk A, Miniszewska J, Pietrzak A, Zalewska-Janowska A. Sense of coherence as a protective factor in chronic urticaria. Postepy Dermatol Alergol. 2017 Abr;34(2):168-73.

21. Rohani C, Abedi H-A, Sundberg K, Langius-Eklöf A. Sense of coherence as a mediator of health-related quality of life dimensions in patients with breast cancer: a longitudinal study with prospective design. Health Qual Life Outcomes [Internet]. 2015 Dic [citado el 2018 Feb 1];13. Disponible en: https://www.ncbi.nlm.nih. gov/pmc/articles/PMC4674962/

22. Suraj S, Singh A. Study of sense of coherence health promoting behavior in north Indian students. Indian J Med Res. 2011 Nov;134(5):645. 
23. Lindmark U, Stegmayr B, Nilsson B, Lindahl B, Johansson I. Food selection associated with sense of coherence in adults. Nutr J. 2005 Feb 28;4:9.

24. Hill R, Tinning R, McCuaig L, Quennerstedt M. Physical Activity and Sense of Coherence in Older Australians. J Aging Phys Act. 2016;24(Suppl.):S111-2.

25. Wainwright NWJ, Surtees PG, Welch AA, Luben RN, Khaw K-T, Bingham SA. Sense of coherence, lifestyle choices and mortality. J Epidemiol Community Health. 2008 Sep 1;62(9):829-31.

26. Wainwright NWJ, Surtees PG, Welch AA, Luben RN, Khaw K, Bingham SA. Healthy lifestyle choices: could sense of coherence aid health promotion? J Epidemiol Community Health. 2007 Out;61(10):871-6.

27. Hilding A, Eriksson A-K, Agardh EE, Grill V, Ahlbom A, Efendic S, et al. The impact of family history of diabetes and lifestyle factors on abnormal glucose regulation in middle-aged Swedish men and women. Diabetologia. 2006 Nov;49(11):2589-98.

28. Kouvonen AM, Väänänen A, Woods SA, Heponiemi T, Koskinen A, Toppinen-Tanner S. Sense of coherence and diabetes: a prospective occupational cohort study. BMC Public Health. 2008 Feb 6;8:46.

29. Peer N, Steyn K, Lombard C, Lambert EV, Vythilingum B, Levitt NS. Rising diabetes prevalence among urban-dwelling black South Africans. PloS One. 2012;7(9):e43336.

30. Nilsen V, Bakke PS, Rohde G, Gallefoss F. Is sense of coherence a predictor of lifestyle changes in subjects at risk for type 2 diabetes? Public Health. 2015 Feb 1;129(2):155-61.

31. Powers MA, Bardsley J, Cypress M, Duker P, Funnell MM, Fischl AH, et al. Diabetes self-management education and support in type 2 diabetes: a joint position statement of the American Diabetes Association, the American Association of Diabetes Educators, and the Academy of Nutrition and Dietetics. Diabetes Educ. 2015;41(4):417-30.

32. Galaviz KI, Narayan KMV, Lobelo F, Weber MB. Lifestyle and the Prevention of Type 2 Diabetes: a Status Report. Am J Lifestyle Med. 2015 Nov 24;12(1):4-20.

33. Márquez-Palacios JH, Urzúa-Morales A, Calderón-Carvajal C, Salazar-Estrada J, Díaz-Reséndiz F. El efecto mediador del sentido de coherencia en la relación entre autocuidado y control glucémico de personas diabéticas. Glob Health Promot. 2021 Feb 11.

34. Richardson A, Adner N, Nordström G. Persons with insulin-dependent diabetes mellitus: acceptance and coping ability. J Adv Nurs. 2001 Mar;33(6):758-63.

35. Márquez-Palacios JH, Yanez-Peñúñuri LY, Salazar-Estrada J. Relación entre sentido de coherencia y diabetes mellitus: una revisión sistemática. Ciênc Saúde Colet. 2020;25(10):3955-67.

36. Feldt T, Lintula H, Suominen S, Koskenvuo M, Vahtera J, Kivimäki M. Structural validity and temporal stability of the 13-item sense of coherence scale: prospective evidence from the population-based HeSSup study. Qual Life Res Int J Qual Life Asp Treat Care Rehabil. 2007 Abr;16(3):483-93.

37. Nilsson KW, Leppert J, Simonsson B, Starrin B. Sense of coherence and psychological well-being: improvement with age. J Epidemiol Community Health. 2010 Abr;64(4):347-52.

38. Saravia JC, Iberico C, Yearwood K. Validation of sense of coherence (SOC) 13-item scale in a peruvian sample. J Behav Health Soc Issues [Internet]. 2014 [citado el 2018 Oct 13];6(2). Disponible en: http://www. redalyc.org/resumen.oa? id=282235731003

39. U.K. Prospective Diabetes Study Group. Effect of intensive blood-glucose control with metformin on complications in overweight patients with type 2 diabetes (UKPDS 34). UK Prospective Diabetes Study (UKPDS) Group. Lancet Lond Engl. 1998 Sep 12;352(9131):854-65.

40. U.K. Prospective Diabetes Study Group. Intensive blood-glucose control with sulphonylureas or insulin compared with conventional treatment and risk of complications in patients with type 2 diabetes (UKPDS 33). UK Prospective Diabetes Study (UKPDS) Group. Lancet Lond Engl. 1998 Sep 12;352(9131):837-53.

41. Hu L, Bentler PM. Cutoff criteria for fit indexes in covariance structure analysis: Conventional criteria versus new alternatives. Struct Equ Model Multidiscip J. 1999 Ene 1;6(1):1-55. 
42. Schreiber JB, Nora A, Stage FK, Barlow EA, King J. Reporting Structural Equation Modeling and Confirmatory Factor Analysis Results: a Review. J Educ Res. 2006 Jul 1;99(6):323-38.

43. Hooper D, Couglan J, Mullen MR. Structural equation modelling: guidelines for determining model fit [Internet]. 2008 [citado el 2018 Oct 30]. Disponible en: https://arrow.dit.ie/libart/4

44. Dominguez-Lara SA. El odds ratio y su interpretación como magnitud del efecto en investigación. Educ Médica. 2018 Ene 1;19(1):65-6.

45. Zugravu C. Sense of coherence and its connections with BMI and weight-related beliefs and attitudes. Int J Collab Res Intern Med Public Health. 2012 Jun 1;4:1131.

46. Bae JP, Lage MJ, Mo D, Nelson DR, Hoogwerf BJ. Obesity and glycemic control in patients with diabetes mellitus: analysis of physician electronic health records in the US from 2009-2011. J Diabetes Complications. 2016 Mar;30(2):212-20.

47. Antonovsky A. The salutogenic model as a theory to guide health promotion. Health Promot Int. 1996 Mar 1;11(1):11-8.

48. Super S, Wagemakers M. E, Picavet HSJ, Verkooijen KT, Koelen MA. Strengthening sense of coherence: opportunities for theory building in health promotion. Health Promot Int. 2016 Dec 1;31(4):869-78.

49. Du T, Yuan G, Zhou X, Sun X. Sex differences in the effect of HbA1c-defined diabetes on a wide range of cardiovascular disease risk factors. Ann Med. 2016;48(1-2):34-41.

50. Mottalib A, Salsberg V, Mohd-Yusof B-N, Mohamed W, Carolan P, Pober DM, et al. Effects of nutrition therapy on $\mathrm{HbA} 1 \mathrm{c}$ and cardiovascular disease risk factors in overweight and obese patients with type 2 diabetes. Nutr J [Internet]. 2018 Abr 7 [citado el 2020 May 27];17. Disponible en: https://www.ncbi.nlm.nih.gov/pmc/articles/ PMC5889846/

51. Boniol M, Dragomir M, Autier P, Boyle P. Physical activity and change in fasting glucose and HbA1c: a quantitative meta-analysis of randomized trials. Acta Diabetol. 2017 Nov;54(11):983-91.

52. Lind M, Pivodic A, Svensson A-M, Ólafsdóttir AF, Wedel H, Ludvigsson J. HbA1c level as a risk factor for retinopathy and nephropathy in children and adults with type 1 diabetes: swedish population based cohort study. BMJ [Internet]. 2019 Ago 28 [citado el 27 de mayo de 2020];366. Disponible en: https://www.bmj.com/ content/366/bmj. 14894

53. Choi D-W, Jeon J, Lee SA, Han K-T, Park E-C, Jang S-I. Association between Smoking Behavior Patterns and Glycated Hemoglobin Levels in a General Population. Int J Environ Res Public Health [Internet]. 2018 Oct [citado el 2020 May 27];15(10). Disponible en: https://www.ncbi.nlm.nih.gov/pmc/articles/PMC6210515/

54. Hernández-Avila M, Garrido F, Salazar-Martínez E. Sesgos en estudios epidemiológicos. Salud Pública México. 2000 Sep;42:438-46.

55. Olsson M, Gassne J, Hansson K. Do different scales measure the same construct? Three Sense of Coherence scales. J Epidemiol Community Health. 2009 Feb 1;63(2):166-7.

56. Eriksson M, Lindström B. Validity of Antonovsky's sense of coherence scale: a systematic review. J Epidemiol Community Health. 2005 Jun;59(6):460-6.

57. Terwee CB, Bot SDM, Boer MR, van der Windt DAWM, Knol DL, Dekker J, et al. Quality criteria were proposed for measurement properties of health status questionnaires. J Clin Epidemiol. 2007 Ene;60(1):34-42.

58. Virués-Ortega J, Martínez-Martín P, Del Barrio JL, Lozano LM, Grupo Español de Estudios Epidemiológicos sobre Envejecimiento. Cross-cultural validation of Antonovsky's Sense of Coherence Scale (OLQ-13) in Spanish elders aged 70 years or more. Med Clin (Barc). 2007 Abr 7;128(13):486-92.

59. Zimprich $D$, Allemand M, Hornung R. Measurement invariance of the abridged sense of coherence scale in adolescents. Eur J Psychol Assess. 2006;22(4):280-7. 
Dirección del primer autor:

Jesús Humberto Márquez-Palacios

Universidad Iberoamericana Torreón

Calzada Iberoamericana 2255

Ejido La Unión, C.P. 27420

Torreón - Coahuila - México

Correo: psichumberto@hotmail.com

Dirección para correspondencia:

José Guadalupe Salazar Estrada

Centro Universitario de los Valles - Universidad de Guadalajara

Carretera Guadalajara - Ameca, km. 45.5

C.P. 46600. Ameca, Jalisco, México

Correo: jsalazar@valles.udg.mx

Cómo citar: Márquez-Palacios JH, Salazar-Estrada JG, Urzúa-Morales A. Asociación entre sentido de coherencia y control glucémico en personas con Diabetes Mellitus tipo 2. Rev Bras Promoç Saúde. 2021;34:11905. 